\title{
G2 to M Transition Process
}

National Cancer Institute

\section{Source}

National Cancer Institute. G2 to M Transition Process. NCI Thesaurus. Code C28563.

Progression from G2 phase to M phase of the standard mitotic cell cycle. (From Gene Ontology) 